\title{
Microbiome profiling reveals distinct microbial compositions of nasal polyps in chronic rhinosinusitis
}

\section{Sung-Woo Cho}

Seoul National University Bundang Hospital https://orcid.org/0000-0003-0827-4471

\section{Dong-Young Kim}

Seoul National University College of Medicine

\section{Sungmi Choi}

Korea University

Hye-Ryun Kang ( $\nabla$ helenmed@snu.ac.kr)

Seoul National University College of Medicine

\section{Hana Yi}

Korea University

\section{Research}

Keywords: biodiversity, microbiota, nasal polyps, Prevotella, sinusitis

Posted Date: August 18th, 2020

DOl: https://doi.org/10.21203/rs.3.rs-58912/v1

License: (9) This work is licensed under a Creative Commons Attribution 4.0 International License. Read Full License 


\section{Abstract}

Background: Chronic rhinosinusitis (CRS) is characterized according to the presence or absence of nasal polyps (NPs) and displays nasal microbiota dysbiosis. However, optimal sampling methods of the nasal microbiome in CRS have not been identified. We therefore aimed to assess the microbial composition in patients with CRS, comparing different sampling methods (swab and tissue biopsy), tissue types (uncinate tissue and NP), and disease subtypes.

Methods: Samples were obtained by swabbing the middle meatus and taking a biopsy of uncinate tissue (UT) in 8 patients with CRS with NP (CRSwNP), 6 patients with CRS without NP (CRSsNP), and 8 controls. NPs were also harvested in CRSwNP. Extracted DNA samples were analyzed by $16 \mathrm{~S}$ rRNA gene amplicon sequencing.

Results: The microbiome diversity did not significantly differ between disease subtypes in nasal swabs or UT samples. However, a principal coordinates analysis based on weighted UniFrac distances revealed a greater interpersonal variance for nasal swabs than for UT. UT samples presented with a distinct pattern and composition in CRSwNP compared to CRSsNP or control. Compared to UT, NP revealed a unique microbiome profile with significantly less bacterial diversity. Prevotella was the most abundant genus in the UT regardless of the disease subtype and its prevalence was significantly reduced in NP. Prevotella abundance was negatively correlated with disease severity as measured by Lund-Mackay scoring.

Conclusion: Tissue samples are better specimens than nasal swabs for assessing the microbiomes of CRS patients. Microbiomes of NP tissues revealed an association with clinical severity of CRSwNP.

\section{Background}

Chronic rhinosinusitis (CRS) is a common inflammatory upper airway disease that affects approximately $12.1 \%$ of the US population (1) and $8.4 \%$ of the Korean population (2). CRS is clinically defined based on the presence of symptoms for over three months, which include positive endoscopic and/or computed tomography (CT) findings. CRS can be further classified according to the presence of nasal polyps (NPs) as CRS with NP (CRSwNP) and CRS without NP (CRSsNP) (3).

Clinically, it is important to differentiate between CRSwNP and CRSsNP, as patients with the former have a greater disease burden than those suffering from the latter in terms of disease severity and poor treatment outcomes $(4,5)$. Additionally, CRSwNP and CRSsNP are known to have distinct biological characteristics, indicating the diverse pathogenesis of CRS as depicted by different inflammatory profiles (6).

Numerous studies have examined the role of commensal bacterial communities in CRS development. Interactions between the host immune system and sinonasal bacteria implicate microbiota in disease pathogenesis. For example, a well-known association exists between the Staphylococcus aureus superantigen IgE and Th2 inflammation in NPs $(7,8)$. Additionally, Pseudomonas aeruginosa is known to 
be associated with a poorer quality of life and unfavorable outcomes in patients with CRS $(9,10)$. However, the roles of other diverse bacteria in the pathogenesis of CRS remain unclear.

Although recent studies of the nasal microbiome have described the overall composition and dysbiosis of the microbiome in CRS (11-14), individual microbial data vary between studies. This discrepancy could be explained by diverse technical approaches and sampling methods utilized in each study, different anatomical sampling sites, and heterogenous patient distribution, such as different proportions of CRSWNP and CRSsNP.

In this study, nasal microbiota were analyzed in patients with CRS according to the sampling method (swab vs. tissue), disease subtype (with or without NPs), and tissue type [uncinate tissue (UT) vs. NP]. Considering the potential role of bacteria in the pathogenesis of CRS and the distinct characteristics of CRSwNP compared to CRSsNP, the microbiome profiles in the NP are likely to have distinct characteristics.

\section{Methods}

\section{Study participants}

This cross-sectional study was based on the nasal microbiome profiles of patients who underwent primary endoscopic sinus surgery at Seoul National University Hospital. CRSWNP and CRSsNP were diagnosed based on the European Position Paper on Rhinosinusitis with NPs (3). All patients were initially managed medically with three weeks of systemic antibiotics and intranasal corticosteroid treatment. Endoscopic sinus surgery was performed if no improvement was observed after initial therapy. Subjects who underwent endoscopic septoplasty for nasal-septal deviation without evidence of CRS were recruited as controls. Systemic steroids and antibiotics were avoided for at least four weeks before surgery.

Demographic variables including age, sex, smoking history, atopy, asthma, antibiotic use, and intranasal steroid administration were evaluated for all patients. Atopy was assessed by either a skin prick test to detect reactions to common inhalant allergens or a multiple allergen simultaneous test (Immunosystems, Mountain View, CA, USA). Patients presenting with a positive response to methacholine inhalation or short-acting $\beta 2$ agonists with a history of wheezing, shortness of breath, or chest tightness were regarded as having asthma (15). Quantification of disease severity was analyzed by CT using the Lund-Mackay (LM) scoring system (16).

\section{Sample collection}

Three different types of samples were acquired: 1) nasal swabs from the middle meatus, 2) tissue biopsied from the uncinate process (UT), and 3) NP tissue biopsied from the ethmoid sinus. All samples were collected intra-operatively. To eliminate the possibility of contamination, surgical draping with a solution composed of $2 \%$ chlorhexidine gluconate and $70 \%$ isopropyl alcohol was used for all patients. Swabs were taken from the middle meatus under endoscopic guidance with cotton ball swabs 
(Transystem ${ }^{\mathrm{T}}$, COPAN, Brescia, Italy). After swabbing, the UT or NP was harvested surgically. All samples were collected by a single surgeon. Samples were immediately separated into sterile containers, placed on ice, and transported to the laboratory for storage at $-80^{\circ} \mathrm{C}$. Any swabs that may have contacted the nasal vestibule were discarded.

\section{DNA extraction, PCR amplification, and 16S rRNA gene sequencing}

Swab heads were thawed, cut into small pieces, and then placed in $180 \mu \mathrm{L}$ of enzymatic lysis buffer (Qiagen, Hilden, Germany) overnight at room temperature. Bead homogenization was performed with 5$\mathrm{mm}$ steel beads agitated for $20 \mathrm{~s}$ at $15 \mathrm{~Hz}$, followed by $0.1-\mathrm{mm}$ glass beads for $5 \mathrm{~min}$ at $30 \mathrm{~Hz}$. The same extraction procedure was carried out for the tissue biopsy samples. The remainder of the extraction protocol was performed using the FastDNA® SPIN Kit for Soil DNA extraction according to the manufacturer's instructions (MP Biomedicals, Irvine, CA, USA). Extracted DNA was stored at $-80{ }^{\circ} \mathrm{C}$ until sequencing. PCR amplification of the V3-V4 hypervariable region of the bacterial 16S rRNA gene was performed using $3 \mu \mathrm{L}$ of the extracted DNA template and $25 \mu \mathrm{L} 2$ X KAPA HiFi HotStart ReadyMix (KAPA Biosystems, Wilmington, MA, USA). The primers 318F (5' TCGTCGGCAGCGTCAGATGTGTATAAGAGACAGCCTACGGGNGGCWGCAG-3') and 806R (5' GTCTCGTGGGCTCGGAGATGTGTATAAGAGACAGGACTACHVGGGTATCTAATCC-3') were used for 16S rRNA gene amplification. The PCR cycling conditions were $3 \mathrm{~min}$ at $95^{\circ} \mathrm{C} ; 25$ cycles for $30 \mathrm{~s}$ at $95^{\circ} \mathrm{C}, 30 \mathrm{~s}$ at $55^{\circ} \mathrm{C}$, and $30 \mathrm{~s}$ at $72{ }^{\circ} \mathrm{C} ; 5 \mathrm{~min}$ at $72{ }^{\circ} \mathrm{C}$; holding at $4{ }^{\circ} \mathrm{C}$. The amplicons were purified using Agencourt AMPure XP magnetic beads (Beckman Coulter, Brea, CA, USA). The purified products were subjected to index PCR using a Nextera Index Kit (Illumina, San Diego, CA, USA) following the manufacturer's instructions. The products were further purified using AMPure XP beads. The resulting bacterial amplicon library was quantified, mixed with multiple libraries, and sequenced using the MiSeq v3 platform (Illumina).

\section{Sequence data processing}

The raw sequence data files were preprocessed using several bioinformatics tools before downstream data analysis. To remove sequences with low quality scores, the raw reads were pre-filtered using PRINSEQ (17). Adapter sequences were removed using CUTADAPT (18) and ambiguous bases were further trimmed using the FASTX-toolkit (19). Pair-end reads were merged using PEAR (20) and further filtered with PRINSEQ. Chimeric sequences and singletons were screened and reduced using USEARCH (21). A random sampling of 10000 reads was performed to equalize the sequencing depth between samples. Downstream data analysis was completed using QIIME (22) with the Ezbiocloud 16S rRNA gene sequence database (21). Operational taxonomic units were defined as clusters of sequences with $\geq 97 \%$ identity. Alpha- and beta-diversity indices were calculated using the core_diversity_analyses option. The weighted normalized UniFrac distance or Bray-Curtis distance was plotted by principal coordinates analysis (PCoA).

\section{Statistical analysis}

Clinical parameters among each group were compared by Kruskal-Wallis tests followed by the MannWhitney U test for post hoc comparison. Alpha diversity metrics among each group were compared using 
the Kruskal-Wallis test, and a Wilcoxon signed rank test was used for paired analyses. $P$ values were adjusted for multiple comparisons using the Benjamini-Hochberg method. The associations between the microbiome compositions and the study groups were tested using permutational multivariate analysis of variance (PERMANOVA). Linear discriminant analysis effect size (LEfSe) (23), a method for biomarker discovery, was used to identify genera that best characterized each study group. Taxa with linear discriminant analysis scores $>2$ and $P<0.05$ were considered to be significant. Simple linear regression was performed to identify the most relevant genera related to disease severity according to the LM score in CT scans. Spearman correlation coefficients were determined to assess the relationships between the relative abundance of genera and disease severity. Statistical analyses were performed using SPSS ver. 22.0 software (SPSS, Inc., Chicago, IL, USA). Simple linear regression was performed using Weka software. ${ }^{24}$ Significance was accepted at $P<0.05$ for multiple analyses.

\section{Results}

\section{Clinical characteristics of study participants}

Samples from 22 adults in 3 different groups (8 CRSwNP patients, 6 CRSsNP patients, and 8 control subjects) were collected. Among the three groups, clinical parameters including age, male to female ratio, presence of atopy or asthma, usage of intranasal steroid or oral antibiotics, and smoking status did not differ, but pre-operative CT scores showed significant differences $(P=0.001$, Table 1$)$. CRS groups had significantly higher CT scores than controls ( $9.9 \pm 5.5$ vs. $0.1 \pm 0.4, P<0.001)$; however, there was no significant difference between the average CT scores of CRSwNP and CRSsNP groups (10.9 \pm 5.1 vs. 8.5 $\pm 6.2, P=0.491)$. 
Table 1

Clinical characteristics of study participants

\begin{tabular}{|lllll|}
\hline & CRSwNP & CRSsNP & Control & P-value \\
\hline $\mathrm{N}$ & 8 & 6 & 8 & - \\
\hline Mean age (years \pm SD) & $46.0 \pm 18.4$ & $39.2 \pm 14.5$ & $45.8 \pm 17.6$ & 0.973 \\
M/F & $3 / 5$ & $5 / 1$ & $4 / 4$ & 0.222 \\
\hline Atopy & $1 / 8$ & $2 / 6$ & $3 / 8$ & 0.493 \\
\hline Asthma & $2 / 8$ & $0 / 6$ & $0 / 8$ & 0.146 \\
\hline Smoking & $1 / 8$ & $1 / 6$ & $0 / 8$ & 0.514 \\
\hline Nasal steroid & $5 / 8$ & $4 / 6$ & $4 / 8$ & 0.797 \\
\hline Preoperative antibiotics (within 6 months) & $3 / 8$ & $3 / 6$ & $1 / 8$ & 0.300 \\
\hline Lund-Mackay score $( \pm$ SD) & $10.9 \pm 5.1$ & $8.5 \pm 6.2$ & $0.1 \pm 0.4$ & 0.001 \\
\hline Blood eosinophil $(\% \pm$ SD) & $4.0 \pm 3.1$ & $4.6 \pm 2.0$ & $3.1 \pm 2.2$ & 0.759 \\
\hline $\begin{array}{l}\text { Abbreviations: CRSwNP, chronic rhinosinusitis with nasal polyp; CRSsNP, chronic rhinosinusitis } \\
\text { without nasal polyp; SD, standard deviation; M, male; F, female. }\end{array}$ & & \\
\hline
\end{tabular}

\section{Microbial composition varies based on sample sites and disease subtypes}

The microbial composition from swabs and UT were compared among the three groups (control, CRSsNP, and CRSwNP; Fig. 1). There was no significant difference in a-diversity (using the Shannon index) as a function of disease status for the swab and UT samples (Supplemental Figure S1, Additional File 1).

At the phylum level, 11 bacterial phyla were detected in the nasal microbiome (Fig. 1A). Bacteroidetes, Firmicutes, Proteobacteria, Actinobacteria, and Fusobacteria were the dominant phyla, comprising more than $>99 \%$ in both swabs and UT regardless of the disease status. The 30 most abundant genera in nasal microbiomes across sampling sites and disease subtypes are presented in Fig. 1B. In general, Prevotella was the most common in both swabs and UT, but the other major abundant taxa differed significantly between sample types; Staphylococcus, Propionibacterium, and Kluyvera were more abundant in swabs and Ralstonia, Ruminococcus and Barnesiella were more abundant in UT (Supplemental Figure S2, Additional File 1).

\section{UT sampling provides homogenous microbial profiling associated with disease subtype}

PCoA was performed based on the weighted UniFrac distances, which considers genus-level operational taxonomic unit data (Fig. 2A). Nasal swab samples showed substantial in-group heterogeneity and microbial characteristics were not associated with specific disease subtypes. In contrast, UT formed a tight PCoA cluster, indicating the relative homogeneity of the microbiome in UT as compared to swabs. 
When the UT was analyzed, a distinctive microbiome structure was observed depending on the disease subtype (Fig. 2B). PERMANOVA using distance matrices revealed a significantly different microbial composition between the UT from patients with CRSwNP (UT_CRWNP) and those from both control subjects (UT_control, $P=0.006$ ) and patients with CRSsNP (UT_CRSsNP, $P=0.003$, Table 2). However, the microbiome of UT_CRSsNP did not significantly differ from that of UT_control according to PERMANOVA $(P=0.335)$. To identify bacterial genera associated with disease subtypes, LEfSe was used to compare bacterial abundances at the genus level. In the UT_CRSwNP, Barnesiella, Terrisporobacter, and Leptotrichia were abundant, whereas the UT_CRSsNP showed higher relative abundances of Sporobacter, Fusobacterium, and Oscillibacter. In the UT_control, Ruminococcus and Paraburkholderia were abundant compared to the CRS group (Fig. 3).

Table 2

Permutational multivariate analysis of variance (PERMANOVA) using distance matrices of microbiomes from uncinate tissue and nasal polyps

Comparison Weighted normalized UniFrac distance ( $P$ value)

\section{Disease subtype}

UT_CRSWNP vs. UT_CRSsNP $\quad 0.003$

UT_CRSWNP vs. UT_Control $\quad 0.006$

UT_CRSsNP vs. UT_Control $\quad 0.335$

\section{Tissue type}

NP vs. UT_CRSwNP $\quad 0.002$

NP vs. UT_Control $\quad 0.002$

Abbreviations: NP, nasal polyp; UT_CRSwNP, uncinate tissue from chronic rhinosinusitis with nasal polyp; UT_CRSsNP, uncinate tissue from chronic rhinosinusitis without nasal polyp.

\section{Characteristic dysbiosis in NP: depletion of Prevotella}

The microbiomes of NP tissue from patients with CRSwNP were compared to those from the UT_CRSWNP and UT_control groups. The Shannon index was significantly decreased in NP compared to UT_ CRSwNP, indicating decreased diversity in the NP (Supplementary Fig S1, Additional File 1). PCoA further revealed a discrete microbiome structure in the NP as compared to the UT_CRSWNP or UT_control microbiomes (Fig. 2A). The PERMANOVA values demonstrated a significantly different microbial composition in the NP than in the UT_CRSwNP $(P=0.002)$ and UT_control $(P=0.002)$ groups (Table 2$)$. LEfSe identified several bacterial genera whose abundances clearly differed between the NP and UT (UT_CRSwNP and UT_control) (Fig. 4). The overall microbial taxa distribution of NP is depicted in Fig. 1. The phylum Firmicutes was remarkably reduced in NP, whereas Proteobacteria were more abundant in NP 
than in UT. In contrast, Cyanobacteria accounted for less than $23.1 \%$ of bacteria in the NP but was less than $0.5 \%$ in the UT_CRSWNP and UT_control. Prevotella was the most predominant genus in the UT_CRSwNP (33.0\%) and UT_control (32.1\%) and was severely depleted to $0.3 \%$ in the NP. In addition, Ralstonia, the second most common genus (16.1\%), was reduced to $5.9 \%$ in the NP. Instead, Sediminibacterium (26.8\%), Cyanobacterium_JQ650114 (22.7\%), Labilithrix (11.8\%), Caulobacter(10.3\%), and Sphingomonas (6.5\%) were the most abundant genera in the NP tissue. In all, this decrease in microbiome diversity is indicative of a clear dysbiosis in NP.

\section{Microbiome composition is associated with disease severity}

A simple linear regression was performed to identify the genera most associated with disease severity using the LM score (Fig. 5). The genera most associated with disease severity differed between tissue and disease subtypes. Prevotella was the genus most strongly inversely correlated to disease severity in NP tissue (Rho $=-0.771, P=0.025)$. However, Lachnospira (Rho $=0.745, P=0.034)$ and Roseburia (Rho = $-0.971, P=0.001$ ) were most associated with disease severity in the UT_CRSWNP and UT_CRSsNP, respectively. Several other genera in NP and UT associated with disease severity are summarized in Supplementary Table 1 (Additional File 1).

\section{Discussion}

This study aimed to characterize the microbiome signature of sinonasal tissues from healthy controls, subjects with CRSsNP, and those with CRSwNP using different collection methods (i.e. nasal swab vs. tissue biopsy) and tissue types (UT vs. NP). We demonstrated that tissue samples may be more optimal specimens for assessing the microbiome of CRS than are nasal swabs. Tissues provided less variation between samples; moreover, in UTs different microbial compositions were associated with disease subtype. In addition, NP tissues had a unique microbiome that was clearly differentiated from UT and revealed a strong association with clinical severity of CRSwNP. To our knowledge, this is the first study to use UT to assess the microbiome of patients with CRS.

An advantage of using UT is the relative simplicity of sample collection without causing significant morbidity. Therefore UT can be easily acquired regardless of disease status. The immunological profile of UT has been demonstrated to differ according to disease status. For instance, the eosinophil cationic protein (ECP) level in UT is strongly correlated with overall disease severity, comorbid asthma, and the risk of polyp recurrence $(25,26)$. Furthermore, specific immunological characteristics of UT in different disease subtypes $(6,27)$ seem to be associated with the distinguishing microbiome profile.

Recent studies of CRS have found dysbiosis of nasal microbiota. Despite an overall decrease of microbial richness, evenness, and diversity in CRS, inconsistencies or biases in previous studies limited the identification of clinically relevant genera or species $(14,28,29)$. Furthermore, an optimal sampling method of the nasal microbiome was not yet determined. To date, most studies have utilized nasal swabs, primarily collecting from the middle meatus. The middle meatus is an intersectional area that shares a common drainage pathway with other paranasal sinuses. Therefore, samples from this area 
may reflect multiple sites, which could produce increased heterogeneity. However, another study comparing microbial compositions from swab samples collected at different anatomical sites from patients with CRS still demonstrated a high interpersonal variation, which outweighed location-specific differences. ${ }^{11}$ Our study found similar results, where a high interpersonal variability of swab samples may obscure the differences among disease subtypes.

Tissue samples from the UT and NP were superior to swabs and demonstrated decreased interpersonal differences within subgroups. This allowed for the identification of discrete tissue-associated microbiomes. The high variation in swab samples could be a function of the vulnerability of the nasal surface microbiome to environmental changes.

Our results are further supported by a previous study that found significantly different bacterial compositions in tissue specimens as compared to swab samples (30). Tissue specimens are more inclusive than swabs, as they incorporate bacterial biofilms that grow on the surface and bacteria that penetrate the mucosal epithelium. This is supported by the observation that tissue samples have a greater biomass than swabs, although non-microbial DNA is present (31). Thus, tissue samples should be given greater attention in the future as a collection method.

In CRS, the airway microbiome composition is associated with inflammatory profiles. For example, CRSwNP accompanied by asthma presented with significantly higher levels of Th2-related cytokines. Simultaneously, there was a higher abundance of Proteobacteria than in patients with CRSwNP without asthma. Furthermore, Corynebacterium, which are members of the phylum Actinobacteria, were more abundant in CRSwNP without asthma (32). There is an upregulation of several important cytokines, such as IL-5, in NP compared to UT regardless of disease subtype $(6,27)$. Therefore, the distinct microbial composition of NP compared to that of UT in our study could be associated with differences in inflammatory profiles.

In the NP, the relative abundance of the phylum Firmicutes was remarkably lower than in the UT, whereas the abundance of the phylum Proteobacteria was higher. Despite the use of different sampling methods, the higher abundance of Proteobacteria than that in controls has been consistently reported. At the genus level, Haemophilus, Escherichia, and Moraxella were highly enhanced in CRSwNP as compared to controls (33-35). Furthermore, in our study, Sphingomonas, a genus in the phylum Proteobacteria, was significantly enriched in the NP as compared to in the UT. In a murine asthma model, glycoproteins from Sphingomonas induced type 2 inflammation via natural killer T cells in an IL-4-, IL-13-, and IL-33dependent manner $(36,37)$. Interestingly, Sphingomonas was found to be more abundant in bronchoalveolar lavage fluid from patients with eosinophil-high asthma than in those with eosinophil-low asthma; this is also related to increased airway hyperresponsiveness to methacholine $(38,39)$.

We also evaluated the severity of CRS using LM scores, a widely used radiological parameter assessed by CT. The score increases with certain markers of disease severity, such as the increasing grade of polyposis, nature of surgery offered (i.e. more extensive surgery), and treatment outcome (16). Correlation 
between genera and disease severity differed by both tissue and disease subtype. This indicates unique interactions of microbiome according to the different microenvironmental conditions.

In the NP microbiome, the genus Prevotella was significantly and inversely correlated with disease severity. Similar findings have been observed in other inflammatory diseases including multiple sclerosis (MS). In that study, the abundance of gut Prevotella was reduced in untreated patients with MS, and treatment with disease-modifying therapy was associated with an increased relative abundance of Prevotella (40). This protective effect of Prevotella appears to be mediated through the induction of CD $4^{+}$ FoxP3 ${ }^{+}$regulatory $\mathrm{T}$ cells $(41)$.

There were several limitations of our study. First, the number of patients was rather small, considering the heterogeneity of CRS. Second, we did not analyze the differences between eosinophilic NP and noneosinophilic NP, which are known to exhibit immunological differences (27). Third, 16S rRNA analysis only reveals the presence of bacteria, but it does not analyze their metabolic activity. Fourth, tissue samples dominated by non-microbial DNA can introduce contamination during amplicon sequencing. Therefore, further validation studies with larger sample sizes are necessary. Future studies should also identify interactions between the tissue microenvironment and microbes in association with immunological profiles.

\section{Conclusion}

Tissue microbiomes in CRS reveal highly clustered profiles with low inter-personal differences, with changes are associated with disease status. Most importantly, we identified a distinct NP microbiome composition as compared to UT. Therefore, tissue samples are encouraged to be used for future evaluation of CRS microbiomes. In all, the altered microbial habitat and strong association with CRS disease severity suggests a distinct tissue microenvironment that requires further study.

\section{List Of Abbreviations}

CRS Chronic rhinosinusitis

CRSwNP Chronic rhinosinusitis with nasal polyp

CRSsNP Chronic rhinosinusitis without nasal polyp

LM Lund-Mackey

NP Nasal polyp

UT Uncinate tissue

PERMANOVA Permutational multivariate analysis of variance 
LEfSe Linear discriminant analysis effect size

\section{Declarations}

\section{Ethics approval and consent to participate}

All methods were carried out in accordance with the ethical standards of the 1964 Declaration of Helsinki and its later amendments. This study was approved by the institutional review board of Seoul National University Hospital (IRB-No. 1702-009-828). All participants and parents of minor participants gave written informed consent.

\section{Consent for publication}

Not applicable

\section{Competing interests}

The authors declare that they have no competing interests

\section{Availability of data and materials}

The datasets generated and/or analysed during the current study are not publicly available due to legal reason in Korea, but are available from the corresponding author on reasonable request.

\section{Funding}

This work was supported by grants 30-2016-0300 and 03-2018-0030 from Seoul National University Hospital and grant 14-2018-031 from Seoul National University Bundang Hospital.

\section{Author contributions}

S.-W.C., D.-Y.K., H.-R.K., and H.Y. designed the clinical study and drafted main manuscript. S.-W.C., D.-Y.K were responsible for patient recruitment and data acquisition. S.C. assisted data analysis and gave critical revision for important intellectual content. All authors approved the final version of the manuscript.

\section{Acknowledgements}

None

\section{References}

1. Blackwell DL, Lucas JW, Clarke TC. Summary health statistics for U.S. adults: national health interview survey. Vital Health Stat 10. 2012;2014(260):1-161. 
2. Ahn JC, Kim JW, Lee CH, Rhee CS. Prevalence and Risk Factors of Chronic Rhinosinusitus, Allergic Rhinitis, and Nasal Septal Deviation: Results of the Korean National Health and Nutrition Survey 2008-2012. JAMA Otolaryngol Head Neck Surg. 2016;142(2):162-7.

3. Fokkens WJ, Lund VJ, Mullol J, Bachert C, Alobid I, Baroody F, et al. EPOS 2012: European position paper on rhinosinusitis and nasal polyps 2012. A summary for otorhinolaryngologists. Rhinology. 2012;50(1):1-12.

4. Toros SZ, Bölükbasi S, Naiboğlu B, Er B, Akkaynak C, Noshari H, et al. Comparative outcomes of endoscopic sinus surgery in patients with chronic sinusitis and nasal polyps. Eur Arch Otorhinolaryngol. 2007;246(9):1003-8.

5. Banerji A, Piccirillo JF, Thawley SE, Levitt RG, Schechtman KB, Kramper MA, et al. Chronic rhinosinusitis patients with polyps or polypoid mucosa have a greater burden of illness. Am J Rhinol. 2007;21(1):19-26.

6. Stevens WW, Ocampo CJ, Berdnikovs S, Sakashita M, Mahdavinia M, Suh L, et al. Cytokines in Chronic Rhinosinusitis. Role in Eosinophilia and Aspirin-exacerbated Respiratory Disease. Am J Respir Crit Care Med. 2015;192(6):682-94.

7. Tomassen P, Vandeplas G, Van Zele T, Cardell LO, Arebro J, Olze H, et al. Inflammatory endotypes of chronic rhinosinusitis based on cluster analysis of biomarkers. J Allergy Clin Immunol. 2016;137(5):1449-56.

8. Van Zele T, Gevaert P, Watelet JB, Claeys G, Holtappels G, Claeys C, et al. Staphylococcus aureus colonization and $\mathrm{IgE}$ antibody formation to enterotoxins is increased in nasal polyposis.=. J Allergy Clin Immunol. 2004;114(4):981-3.

9. Bendouah Z, Barbeau J, Hamad WA, Desrosiers M. Biofilm formation by Staphylococcus aureus and Pseudomonas aeruginosa is associated with an unfavorable evolution after surgery for chronic sinusitis and nasal polyposis. Otolaryngol Head Neck Surg. 2006;134(6):991-6.

10. Cleland EJ, Bassiouni A, Wormald PJ. The bacteriology of chronic rhinosinusitis and the preeminence of Staphylococcus aureus in revision patients. Int Forum Allergy Rhinol. 2013;3(8):642-6.

11. Ramakrishnan VR, Gitomer S, Kofonow JM, Robertson CE, Frank DN. Investigation of sinonasal microbiome spatial organization in chronic rhinosinusitis. Int Forum Allergy Rhinol. 2017;7(1):16-23.

12. Biswas K, Hoggard M, Jain R, Taylor MW, Douglas RG. The nasal microbiota in health and disease: variation within and between subjects. Front Microbiol. 2015(9):134.

13. Choi EB, Hong SW, Kim DK, Jeon SG, Kim KR, Cho SH, et al. Decreased diversity of nasal microbiota and their secreted extracellular vesicles in patients with chronic rhinosinusitis based on a metagenomic analysis. Allergy. 2014;69(4):517-26.

14. Lal D, Keim P, Delisle J, Barker B, Rank MA, Chia N, et al. Mapping and comparing bacterial microbiota in the sinonasal cavity of healthy, allergic rhinitis, and chronic rhinosinusitis subjects. Int Forum Allergy Rhinol. 2017;7(6):561-9.

15. Sistek D, Tschopp JM, Schindler C, Brutsche M, Ackermann-Liebrich U, Perruchoud AP, et al. Clinical diagnosis of current asthma: predictive value of respiratory symptoms in the SAPALDIA study. Swiss 
Study on Air Pollution and Lung Diseases in Adults. Eur Respir J. 2001;17(2):214-9.

16. Hopkins C, Browne JP, Slack R, Lund V, Brown P. The Lund-Mackay staging system for chronic rhinosinusitis: how is it used and what does it predict? Otolaryngol Head Neck Surg. 2007;137(4):555-61.

17. Schmieder R, Edwards R. Quality control and preprocessing of metagenomic datasets. Bioinformatics. 2011;27(6):863-4.

18. Martin M. Cutadapt removes adapter sequences from high-throughput sequencing reads. EMBnet J. 2011;17(1):10-2.

19. Gordon A, Hannon GJ. FastX Toolkit 2010 [Available from: http://hannonlab.cshl.edu/fastx_toolkit/.

20. Zhang J, Kobert K, Flouri T, Stamatakis A. PEAR: a fast and accurate Illumina Paired-End reAd mergeR. Bioinformatics. 2014;30(5):614-20.

21. Chun J, Lee JH, Jung Y, Kim M, Kim S, Kim BK, et al. EzTaxon: a web-based tool for the identification of prokaryotes based on 16S ribosomal RNA gene sequences. Int J Syst Evol Microbiol. 2007;51(Pt 10):2259-61.

22. Caporaso JG, Kuczynski J, Stombaugh J, Bittinger K, Bushman FD, Costello EK, et al. QIIME allows analysis of high-throughput community sequencing data. Nat Methods. 2010;7(5):335-6.

23. Segata N, Izard J, Waldron L, Gevers D, Miropolsky L, Garrett WS, et al. Metagenomic biomarker discovery and explanation. Genome Biol. 2011;12(6):R60.

24. Frank E, Hall MA, Witten IH, Workbench TW. Online appendix for "data mining: practical machine learning tools and techniques". 4 ed. Burlington: Morgan Kaufmann; 2016.

25. Min JY, Ocampo CJ, Stevens WW, Price CPE, Thompson CF, Homma T, et al. Proton pump inhibitors decrease eotaxin-3/CCL26 expression in patients with chronic rhinosinusitis with nasal polyps: Possible role of the nongastric H,K-ATPase. J Allergy Clin Immunol. 2017;139(1):130-41.

26. Weibman AR, Huang JH, Stevens WW, Suh LA, Price CPE, Lidder AK, et al. A prospective analysis evaluating tissue biopsy location and its clinical relevance in chronic rhinosinusitis with nasal polyps. Int Forum Allergy Rhinol. 2017;7(11):1058-64.

27. Kim DK, Eun KM, Kim MK, Cho D, Han SA, Han SY, et al. Comparison Between Signature Cytokines of Nasal Tissues in Subtypes of Chronic Rhinosinusitis. Allergy Asthma Immunol Res. 2019;11(2):20111.

28. Abreu NA, Nagalingam NA, Song Y, Roediger FC, Pletcher SD, Goldberg AN, et al. Sinus microbiome diversity depletion and Corynebacterium tuberculostearicum enrichment mediates rhinosinusitis. Sci Transl Med. 2012;4(151):151ra24.

29. Ramakrishnan VR, Hauser LJ, Feazel LM, Ir D, Robertson CE, Frank DN. Sinus microbiota varies among chronic rhinosinusitis phenotypes and predicts surgical outcome. J Allergy Clin Immunol. 2015;136(2):334-42.

30. Kim RJ, Biswas K, Hoggard M, Taylor MW, Douglas RG. Paired analysis of the microbiota of surface mucus and whole-tissue specimens in patients with chronic rhinosinusitis. Int Forum Allergy Rhinol. 
2015;5(10):877-83.

31. Findley K, Oh J, Yang J, Conlan S, Deming C, Meyer JA, et al. Topographic Diversity of Fungal and Bacterial Communities in Human Skin. Nature. 2013;498(7454):367-70.

32. Chalermwatanachai T, Vilchez-Vargas R, Holtappels G, Lacoere T, Jáuregui R, Kerckhof FM, et al. Chronic rhinosinusitis with nasal polyps is characterized by dysbacteriosis of the nasal microbiota. Sci Rep. 2018;8(1):7926.

33. Taylor SL, Leong LEX, Choo JM, Wesselingh S, Yang IA, Upham JW, et al. Inflammatory phenotypes in patients with severe asthma are associated with distinct airway microbiology. J Allergy Clin Immunol. 2018;141(1):94-103.e15.

34. Jain R, Hoggard M, Zoing M, Jiang Y, Biswas $K$, Taylor MW, et al. The effect of medical treatments on the bacterial microbiome in patients with chronic rhinosinusitis: a pilot study. Int Forum Allergy Rhinol. 2018;8:890-9.

35. Copeland E, Leonard K, Carney R, Kong J, Forer M, Naidoo Y, et al. Chronic Rhinosinusitis: Potential Role of Microbial Dysbiosis and Recommendations for Sampling Sites. Front Cell Infect Microbiol. 2018;8:57.

36. Kim HY, Chang YJ, Subramanian S, Lee HH, Albacker LA, Matangkasombut P, et al. Innate lymphoid cells responding to IL-33 mediate airway hyperreactivity independently of adaptive immunity. J Allergy Clin Immunol. 2012;129(1):216-27.

37. Meyer EH, Goya S, Akbari O, Berry GJ, Savage PB, Kronenberg M, et al. Glycolipid activation of invariant T cell receptor+ NK T cells is sufficient to induce airway hyperreactivity independent of conventional CD4+ T cells. Proc Natl Acad Sci U S A. 2006;103(8):2782-7.

38. Sverrild A, Kiilerich P, Brejnrod A, Pedersen R, Porsbjerg C, Bergqvist A, et al. Eosinophilic airway inflammation in asthmatic patients is associated with an altered airway microbiome. J Allergy Clin Immunol. 2017;140(2).

39. Huang YJ, Nelson CE, Brodie EL, Desantis TZ, Baek MS, Liu J, et al. Airway microbiota and bronchial hyperresponsiveness in patients with suboptimally controlled asthma. J Allergy Clin Immunol. 2011;127(2):372-81.

40. Jangi S, Gandhi R, Cox LM, Li N, von Glehn F, Yan R, et al. Alterations of the human gut microbiome in multiple sclerosis. Nat Commun. 2016;7:12015.

41. Mangalam A, Shahi SK, Luckey D, Karau M, Marietta E, Luo N, et al. Human Gut-Derived Commensal Bacteria Suppress CNS Inflammatory and Demyelinating Disease. Cell Rep. 2017;20(6):1269-77.

\section{Figures}


(a)

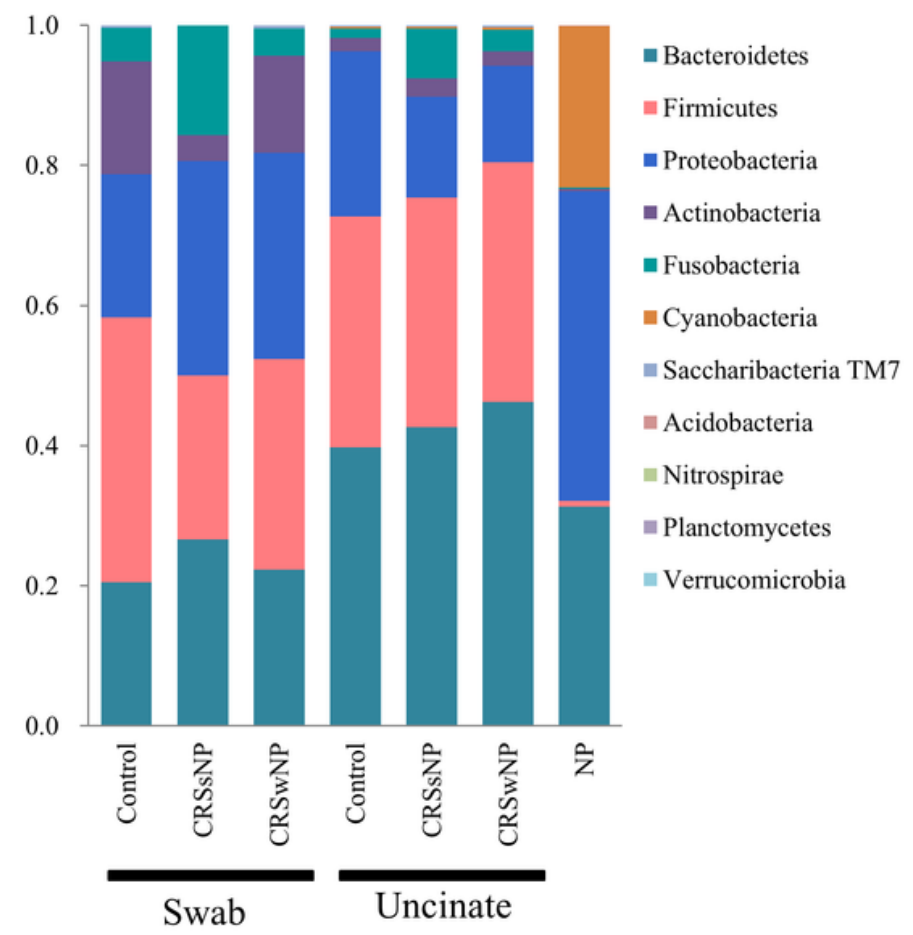

(b)

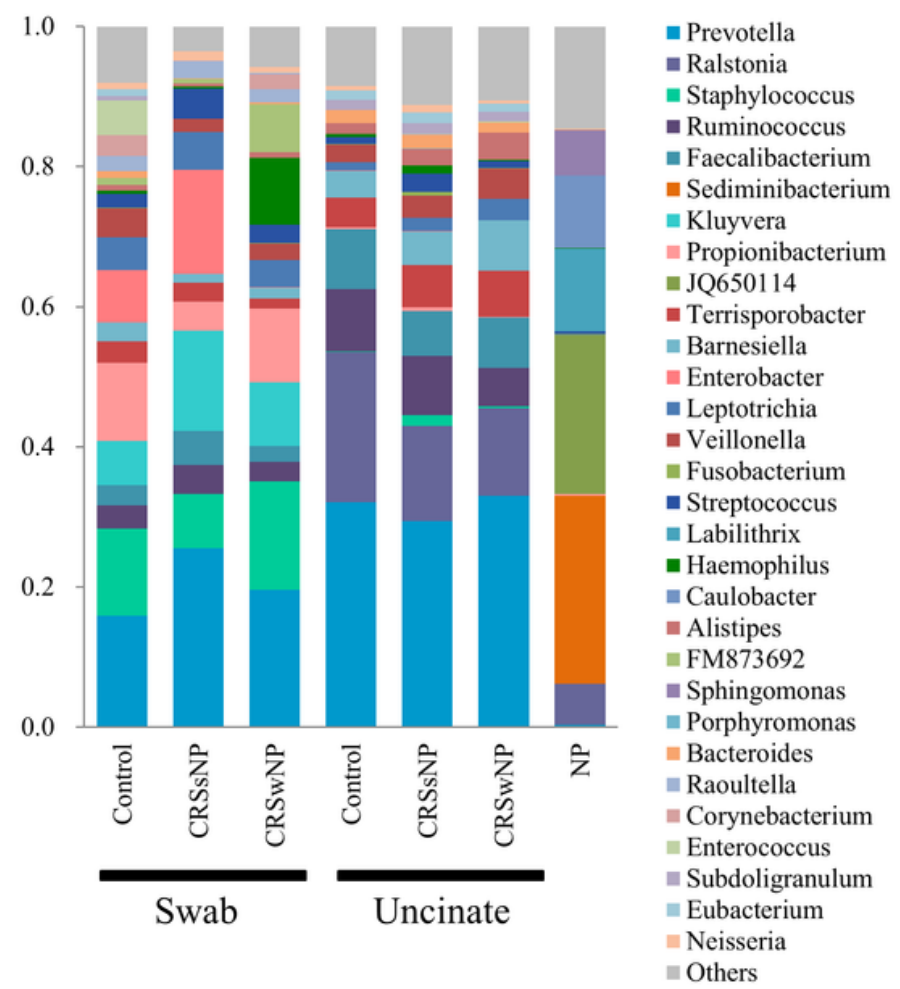

Figure 1

Distribution of bacterial taxa across disease and sampling subsites. Each stacked bar chart summarizes the percent relative abundances. A, At the phylum level, Bacteroidetes, Firmicutes, Proteobacteria, Actinobacteria, and Fusobacteria were the five dominant phyla in both swabs and uncinate tissues regardless of the disease subtype. $B$, Top 30 bacterial genera in the microbiomes according to sample and disease subtypes. Abbreviations: NP, nasal polyp; CRSwNP, chronic rhinosinusitis with nasal polyp; CRSsNP, chronic rhinosinusitis without nasal polyp. 
(a)

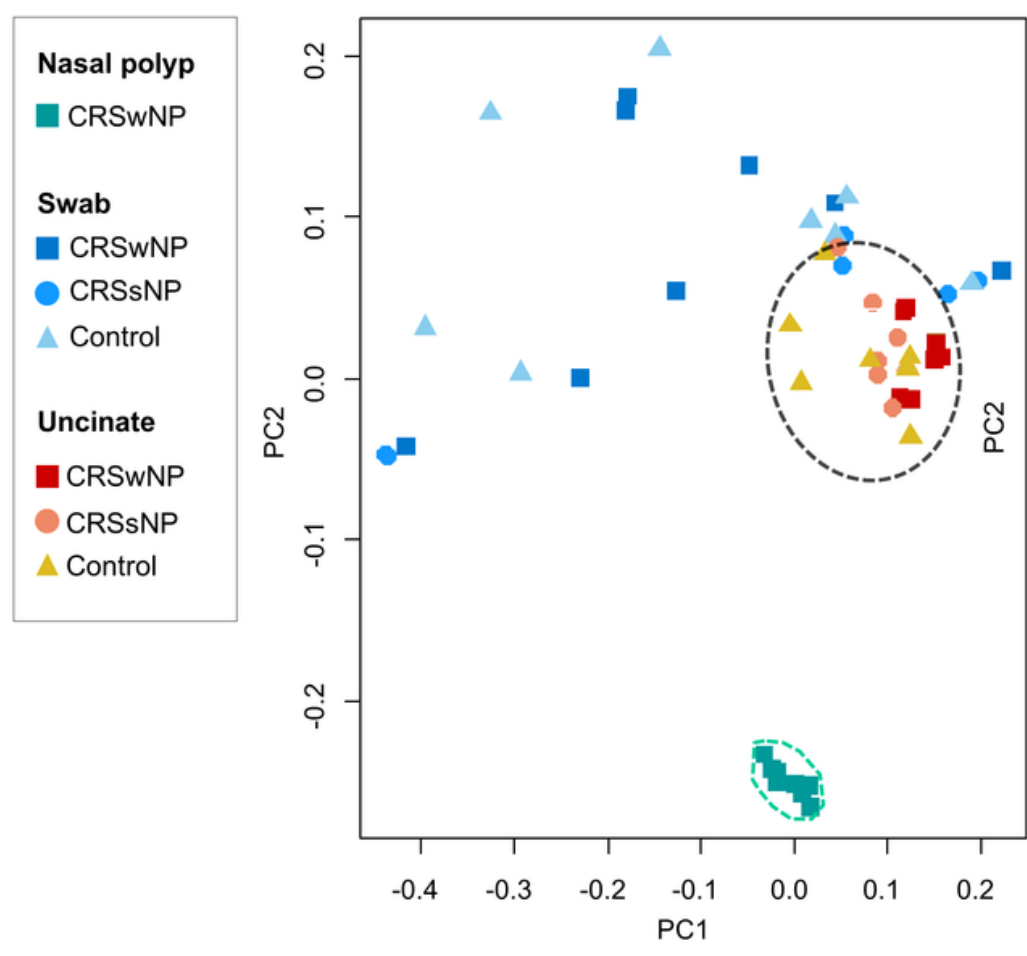

(b)

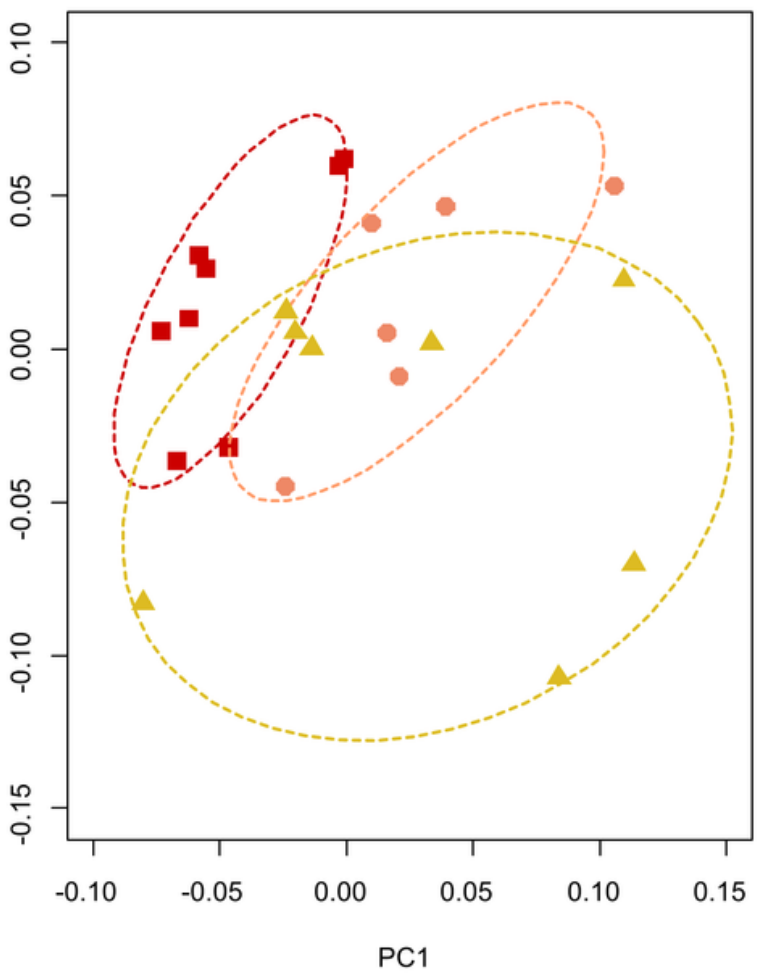

\section{Figure 2}

Variable microbiome structure as a function of clinical sample type and disease subtype. Beta-diversity was calculated by weighted normalized UniFrac distance and PCoA. A, Microbiome distinctiveness depending on the clinical sample type. B, Uncinate tissue microbiome distinctiveness depending on the disease subtype. Confidence ellipse (dotted line) defines the region that contains $68 \%$ of all samples in each group. Abbreviations: CRSwNP, chronic rhinosinusitis with nasal polyp; CRSsNP, chronic rhinosinusitis without nasal polyp. 


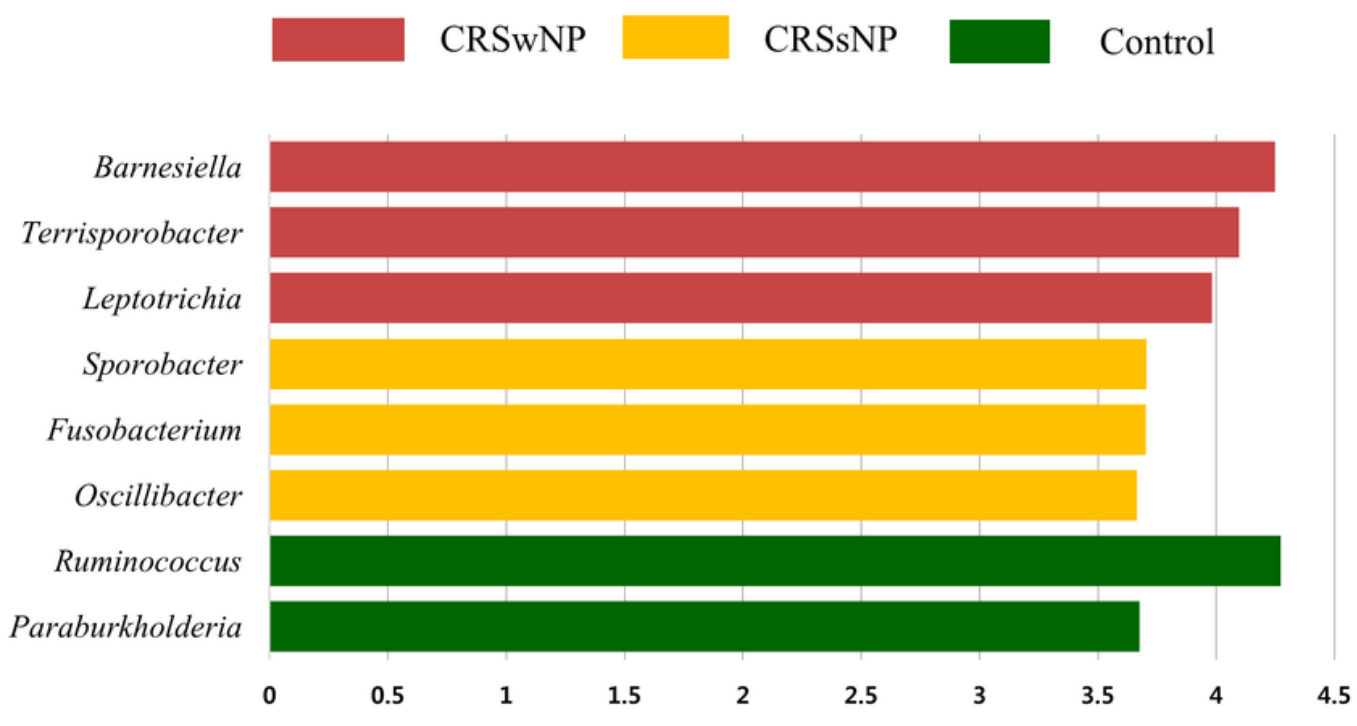

LDA SCORE $(\log 10)$

\section{Figure 3}

Bacterial genera abundances in uncinate tissue differs with disease subtype. Linear discriminant analysis effect size (LEfSe) was used to determine the most abundant genera in CRSWNP, CRSsNP, and controls. Only genera with $\mathrm{P}<0.05$ and LDA score $>2$ are shown. Abbreviations: CRSwNP, chronic rhinosinusitis with nasal polyp; CRSsNP, chronic rhinosinusitis without nasal polyp. 

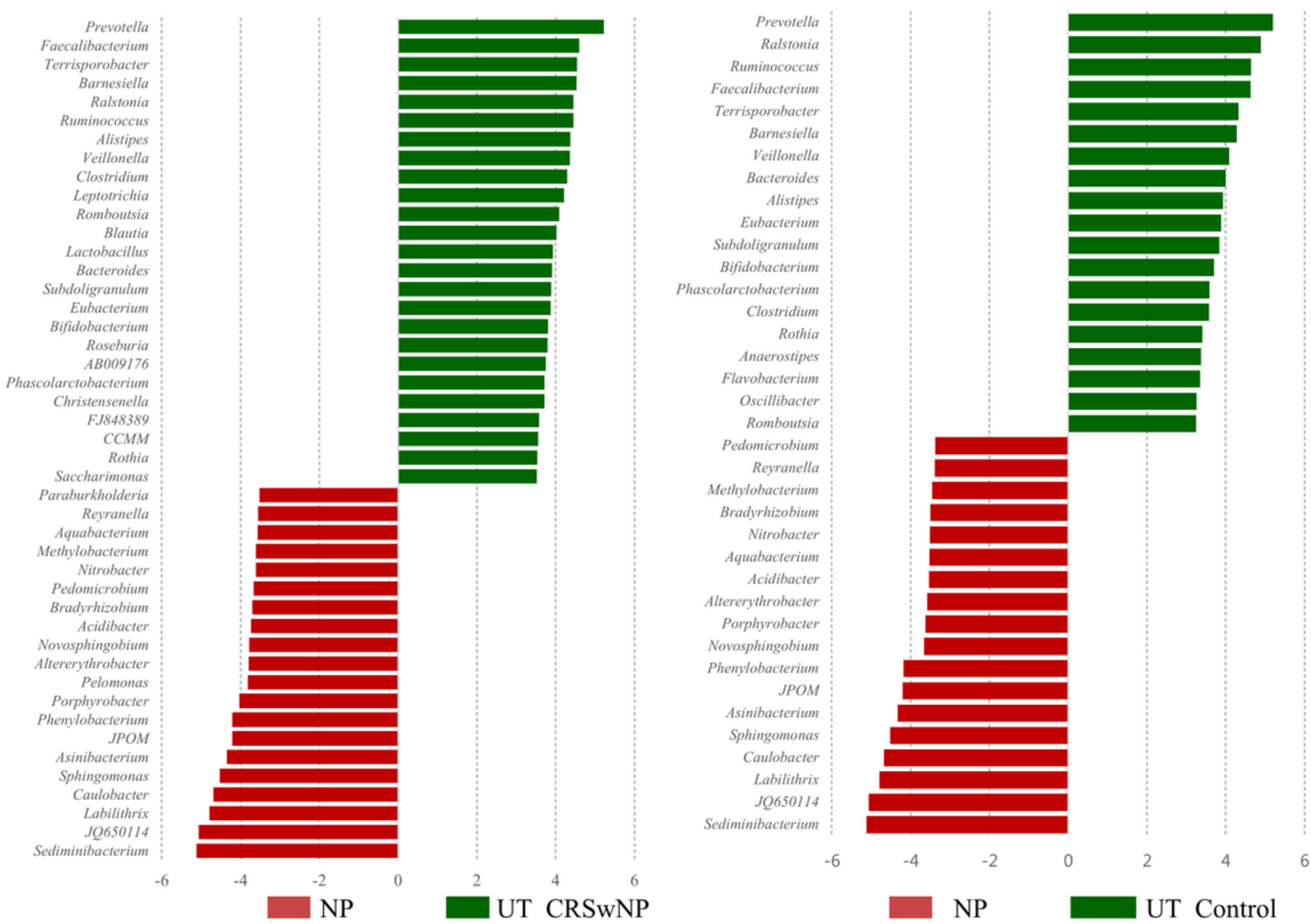

Figure 4

Linear discriminant analysis (LDA) demonstrates distinct bacterial genera between the nasal polyp and uncinate tissue. A, Nasal polyp vs. uncinate tissue from patients with chronic rhinosinusitis with nasal polyp (UT_CRSwNP) and B, nasal polyp vs. uncinate tissue from controls (UT_control). 

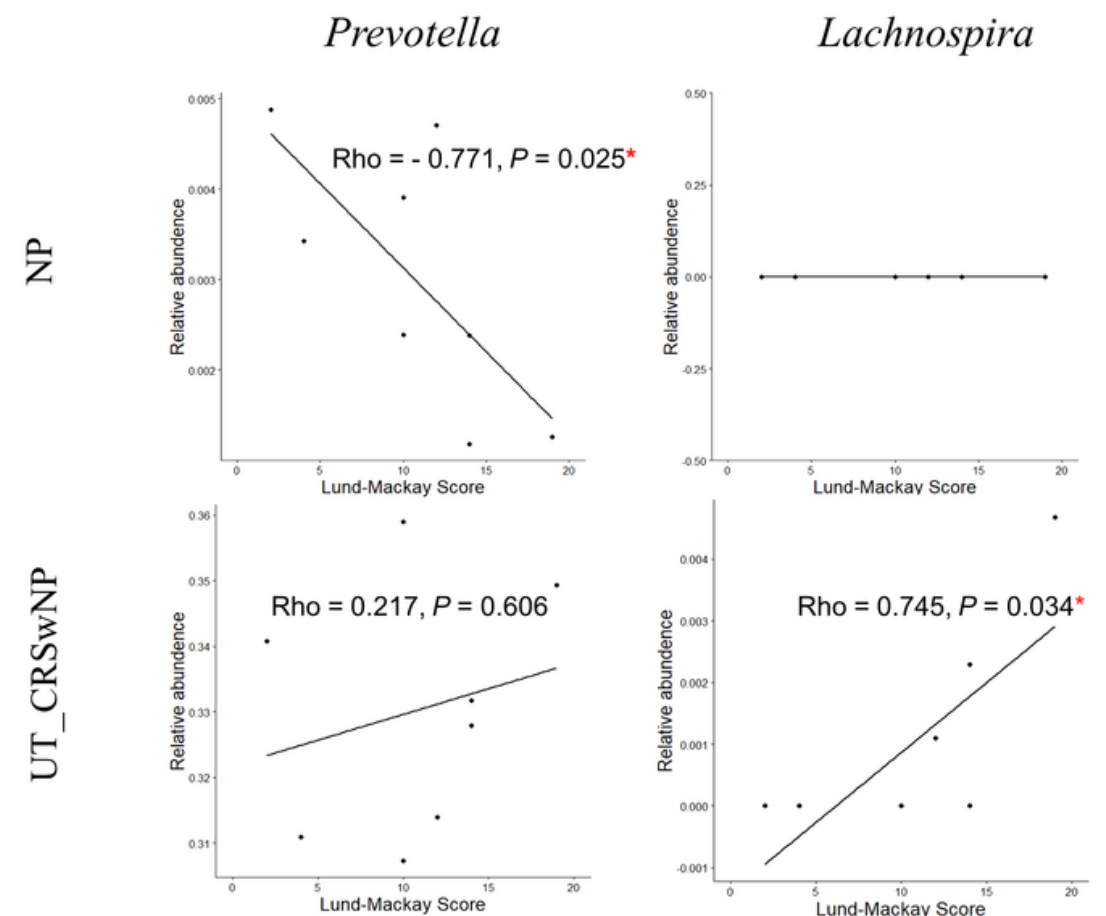

Roseburia
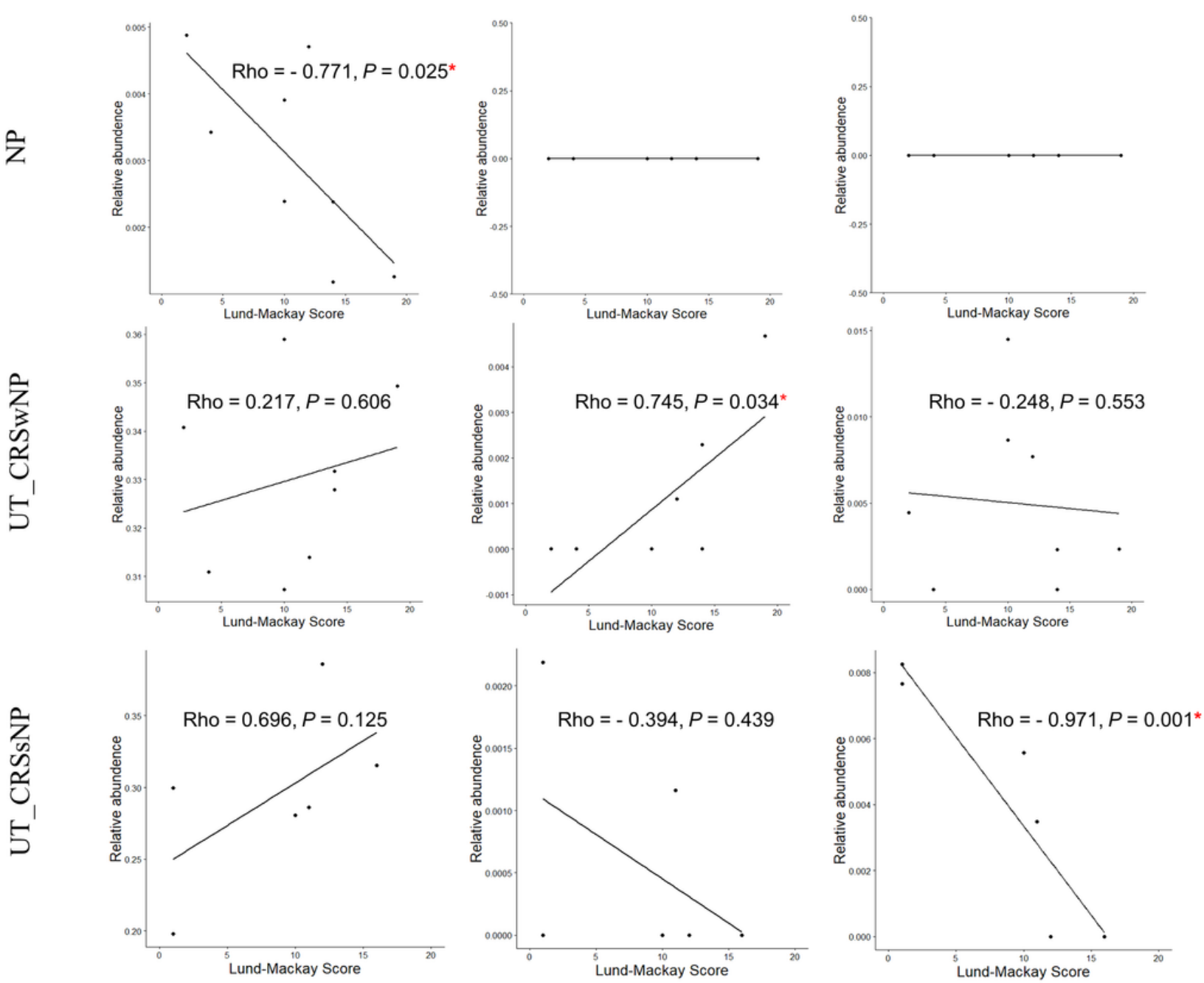

\section{Figure 5}

Correlation between bacterial genera and disease severity. The genera most correlated with disease severity in NP, UT_CRSwNP, and UT_CRSsNP were Prevotella, Lachnospira, and Roseburia, respectively. Relative abundances of these genera were then compared to Lund-Mackay scores by correlation analysis in different tissue microenvironments (NP, UT_CRSwNP, and UT_CRsNP). Abbreviations: NP, nasal polyp; UT, uncinate tissue; CRSwNP, chronic rhinosinusitis with nasal polyp; CRSsNP, chronic rhinosinusitis without nasal polyp; LM, Lund-Mackey.

\section{Supplementary Files}

This is a list of supplementary files associated with this preprint. Click to download.

- Additionalfile1.docx 